\title{
The mechanical property analysis of the large supportive stage with super smooth surface
}

\author{
YueHua Li , BaoShan Zhao and Yong Yang \\ Tianjin Institute of Aerospace Mechanical and Electrical equipment, Tianjin 300045, P R China
}

\begin{abstract}
With the rapid development of satellite astronomy technique, In order to ensure the effective operation of the spacecraft in orbit, the spacecraft requires full ground physical simulation tests. So the construction of the ground simulation test system is an important guarantee for the development of space technology [1]. In the system of full physical simulation test for lunar orbit rendezvous and docking, the large supportive stage with super smooth surface is one of the most important large-scale precision test equipment, this stage can provide a high precision horizontal support surface in the range of $40 \mathrm{~m} * 30 \mathrm{~m}$ for the test load, Each test load is usually supported by three circular air cushion, whose diameter is $200 \mathrm{~mm} \sim 400 \mathrm{~mm}$. High pressure gas is stored in the load equipment, and is released into the air through the air holes on the air cushion, A thin layer of air film is formed between the air cushion and the stage, the thickness of gas film is $10 \mu \mathrm{m} \sim 15 \mu \mathrm{m}$, The load equipment can freely move on the surface of a large stage with the gas film. There are strict requirements for the deformation of the system, Since the floating height is $10 \mu \mathrm{m}$ and the test load is $3 \mathrm{~T}$. So the altitude difference of neighboring platforms is in range of $10 \mu \mathrm{m}$. In order to guarantee the experiment effects, the paper analyses mechanical properties of the stage, distribute index of $10 \mu \mathrm{m}$, and provide theoretical support for subsystem [2], [3].
\end{abstract}

\section{Introduction}

In order to ensure construction requirements of the GNC laboratory, and consider great difference of the Mechanics environment between ground and orbit, the microgravity simulation system has been developed. The spacecraft performance of Mechanics, machinery, navigation and control can be tested and verified [4].

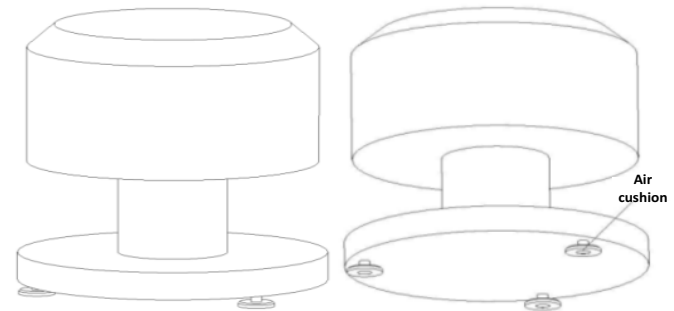

Figure 1. The schematic diagram of test load structure

A supportive surface of high-precision and highlevelness can be provided on the large supportive stage with super smooth surface [5]. Each test load is usually supported by three circular air cushion, whose diameter is $200 \mathrm{~mm} \sim 400 \mathrm{~mm}$, The air cushions are distributed on the circumference of Diameter is $1 \sim 3 \mathrm{~m}$,As shown in Fig. 1. High pressure gas is stored in the load equipment, and is released into the air through the air holes on the air cushion, A thin layer of air film is formed between the air cushion and the stage, the thickness of gas film is $10 \mu \mathrm{m}$ $\sim 15 \mu \mathrm{m}$, The load equipment can freely move on the surface of a large stage with the gas film. As shown in
Fig. 2. The weight of each test load is $3 t$, multiple loads can move freely on surface of the stage with the gas film Under the condition of no friction almost [6]. As shown in Fig. 3.

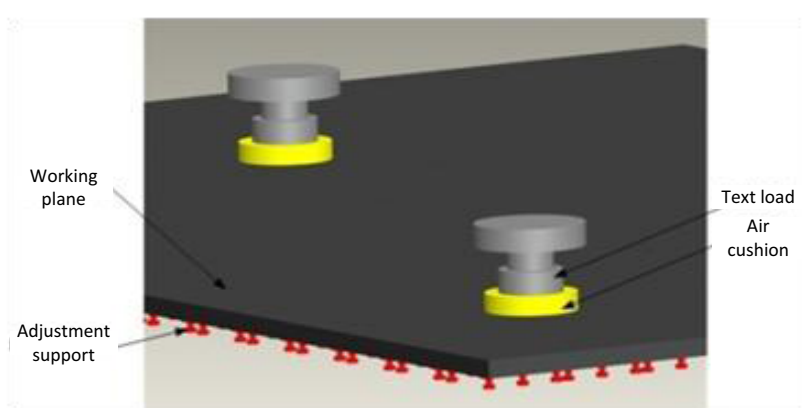

Figure 2. The side view of the large stage

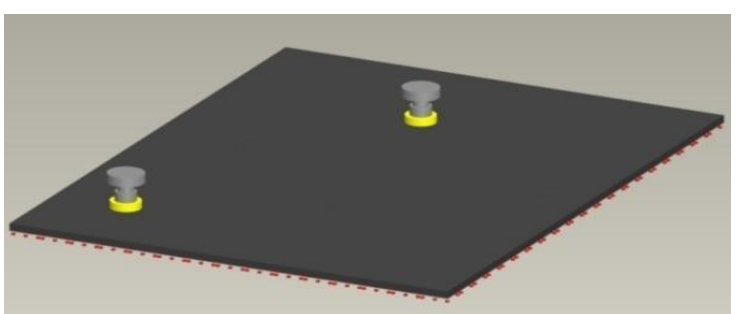

Figure 3. The global graph of the large stage

The large stage is stitched together by 200 block "jinan green" Marble, whose size is $3 \mathrm{~m}^{*} 2 \mathrm{~m} * 0.4 \mathrm{~m}$. Because a plane can be determined by three points, the Supporting method of single block platform is Three 
main supports and three auxiliary supports [7].as shown in Fig. 4. The main supports are 1,3,5, the auxiliary supports are $2,4,6$.

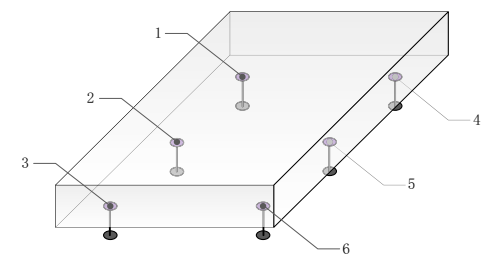

Figure 4. The diagram of supporting method

\section{The subsystems of the large stage}

The large is constituted by six subsystems. Respectively as (1)platform subsystem.(2)foundation subsystem. (3)assemblage subsystem. (4) testing subsystem (5)support and adjustment subsystem (6) integrated control subsystem.as shown in Fig. 5.

The functions of subsystems are as follows:

(1) The material of platform subsystem is "jinan green" marble. A high-levelness and High flatness surface can be provided by the platform, which is the controlled object by integrated control subsystem;

(2) The high grade cement is used to pour foundation subsystem, which is important to satisfy requirement of specifications.

(3) The assemblage subsystem includes method of assembling 200 platforms marble by using specialized car.

(4) The testing subsystem includes method and instrument of testing levelness and altitude difference.

(5) The support and adjustment subsystem includes some test devices, which is used to detect levelness and altitude difference.

(6) The integrated control subsystem includes driving equipment, control algorithm and software.

\section{The index decomposition of altitude difference}

The altitude difference of neighboring platforms is in range of $10 \mu \mathrm{m}$ when the weight of load is $3 \mathrm{t}$.the altitude difference comes from two points as follows:

(1) The adjustment error of mechanical drive is defined as $H_{c}$.

(2) the elastic deformation of overall system is defined as $H_{k}$.

In the following, these two parts above would be analysed, as shown in Fig. 5, and the equation 1 is true.

$$
H=H_{C}+H_{K}
$$

\subsection{The analysis of adjustment error}

The adjustment error of mechanical drive caused by the two parts: one is the accuracy of integrated control subsystem, denoted by $\mathrm{H}_{\mathrm{cp}}$, the other is the Straightness of platform, denoted by $\mathrm{H}_{\mathrm{cl}}$, as shown in Fig. 6, and The equations 2 is true.

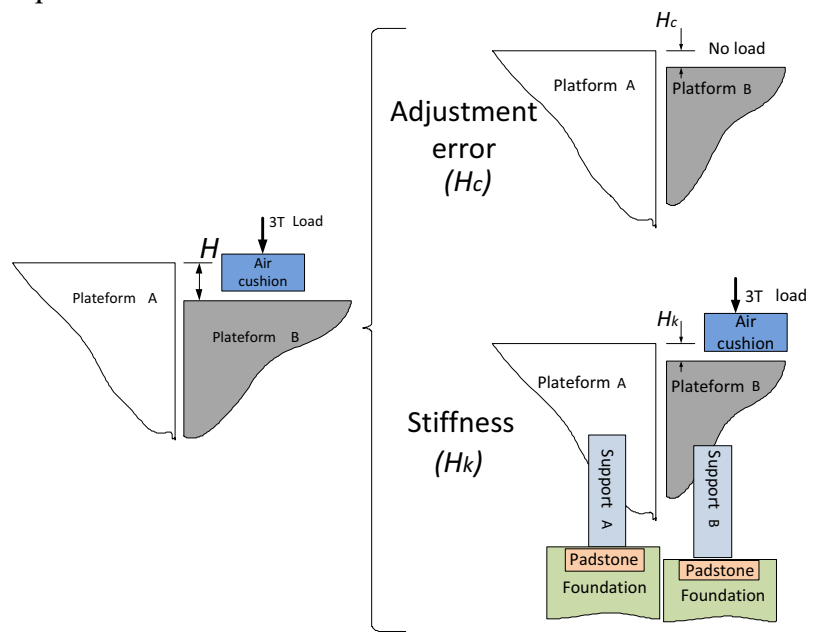

Figure 5. The diagram of decomposing altitude difference

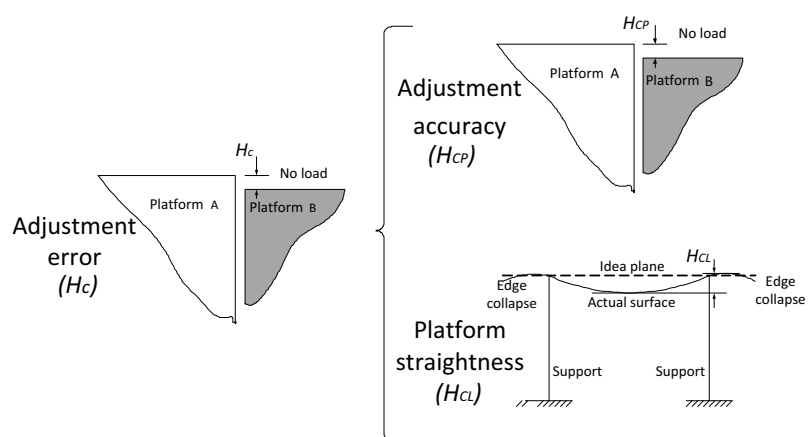

Figure 6. the diagram of decomposing adjustment error

$$
H_{C}=H_{C P}+H_{C L}
$$

\subsubsection{The analysis of adjustment accuracy}

The adjusting methods is that a platform is adjusted without load. so this adjustment accuracy is the accuracy without load.

Based on 3 points leveling method, the distance between support and the edge of platform is small. The speed of adjusting platform is slow, the Height adjustment of support is approximately equal to the lifting height of neighboring platforms. Basis of analysis and research, the adjustment accuracy of servo motor can reach $0.03^{\circ}$. With pienty of experiments and data analysis, the adjustment accuracy of altitude difference can reach $0.2 \mu \mathrm{m}$. The following equation 3 is established.

$$
H_{C P}=0.2 \mu m
$$

\subsubsection{The analysis of platform straightness}

Considering the impact of machining 、 grinding 、 deformation and temperature. Because the impact of straightness, the altitude difference of neighbouring platforms is random. the straightness is got by statistical method usually.

In general, the overall profile is concave when the grinding of platform is finished. as shown in Fig. 7. 
Because of the higher technical targets of altitude difference, the straightness of platform must be no greater than $4 \mu \mathrm{m}$, and satisfy consistence of neighboring platforms.

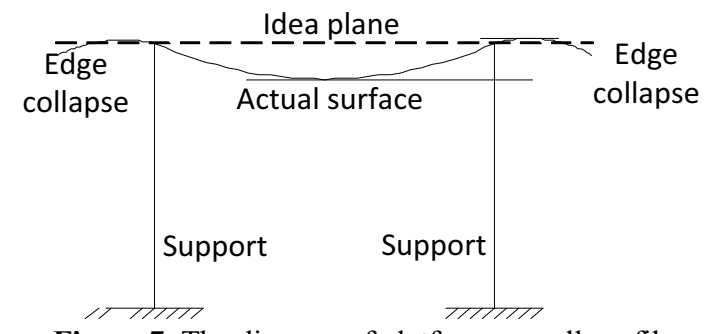

Figure 7. The diagram of platform overall profile

Considering the shape of the overall profile and the effects of temperature, the straightness of platform can reach $3.3 \mu \mathrm{m}$ by test in constant temperature room. The following equation 4 is established.

$$
H_{C L}=3.3 \mu m
$$

Through above analysis, the altitude difference of neighbouring platforms, importing data into equation 2 , and the equation 5 is true.

$$
H_{c}=H_{C P}+H_{C L}=0.2+3.3=3.5 \mu m
$$

\subsection{The analysis of stiffness}

the stiffness of system has effects on altitude difference, include platform stiffness, support stiffness and foundation stiffness.as shown in Fig. 8, and the equation 6 is established.

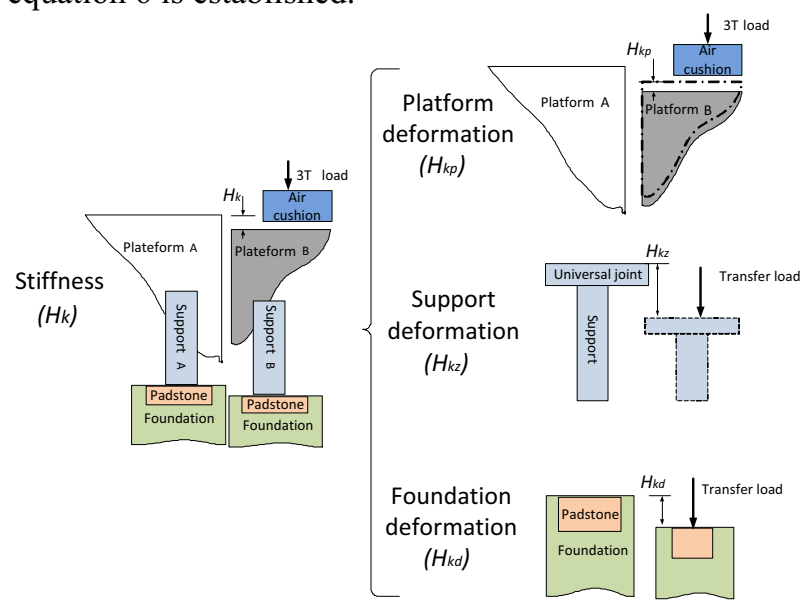

Figure 8. The diagram of index decomposition because of

$$
\begin{gathered}
\text { stiffness } \\
H_{K}=H_{K P}+H_{K Z}+H_{K D}
\end{gathered}
$$

\subsubsection{The analysis of platform mechanical characteristics}

The elasticity deformation of platform is different when the position of air bearing pad is different. As shown in figure 9, the worst condition can be got by calculation and analysis. In the worst condition, the $3 \mathrm{t}$ load can result in maximum deformation of platform. The deformation could be calculated by using finite element method [8], as shown in Fig. 10.

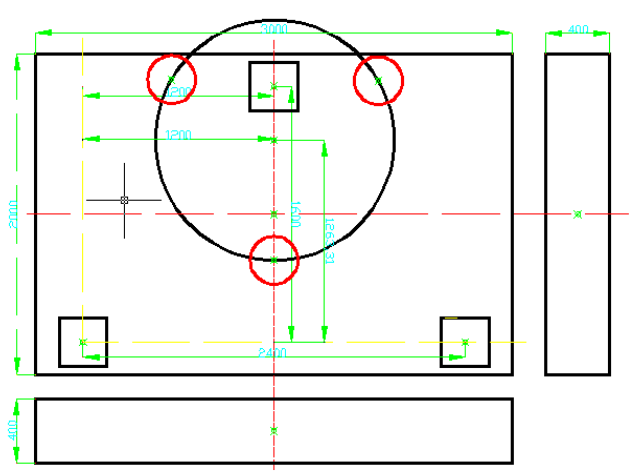

Figure 9.The diagram of the worst condition

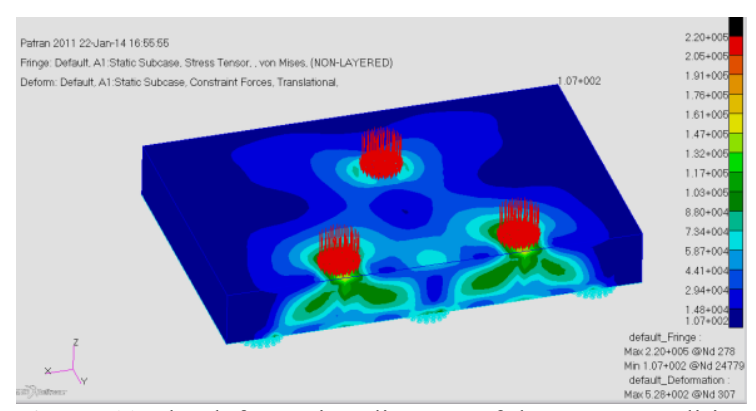

Figure 10.The deformation diagram of the worst condition

Through above analysis, the maximum deformation is $2.2 \mu \mathrm{m}$ when the load is $3 \mathrm{t}$. The following equation 7 is established.

$$
H_{K P}=2.2 \mu m
$$

\subsubsection{The mechanical characteristics analysis of foundation subsystem}

In general, the weight of whole system and the $3 \mathrm{t}$ load would result in the deformation of foundation subsystem. The altitude difference is the most important, the paper would analyze foundation deformation, which is caused by $3 \mathrm{t}$ load. In the process of moving load, the maximum load variation of supporting mechanism can be got by a lot of calculation, this maximum value is the input condition of analyzing foundation deformation. The variation of support 5 is the maximum value, as shown in Fig. 9.

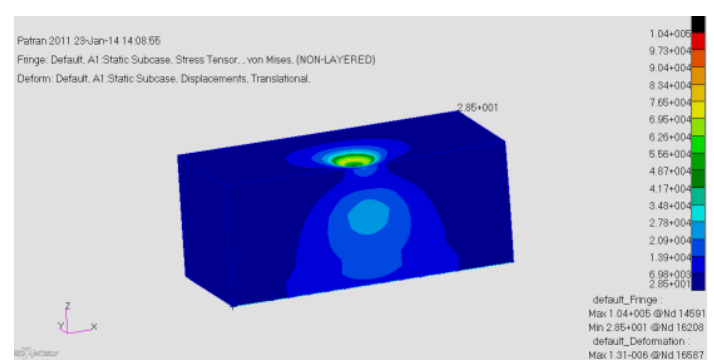

Figure 11. The diagram of the foundation deformation without

$$
\text { steel plate }
$$

Using finite element method, the maximum variation value of support 5 is $12076.6 \mathrm{~N}$. The maximum elastic deformation of foundation subsystem is not more than 
$1.3 \mu \mathrm{m}$ without steel plate. The maximum elastic deformation is not more than $0.98 \mu \mathrm{m}$ with steel plate, and the maximum stress is not more than $0.146 \mathrm{MPa}$, the safety factor is not less than 100. As shown in Fig. 11 and Fig. 12. The following equation 8 is established.

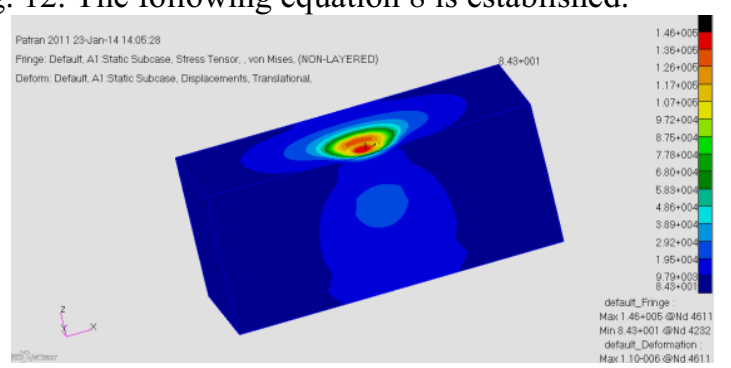

Figure 12. The diagram of the foundation deformation with

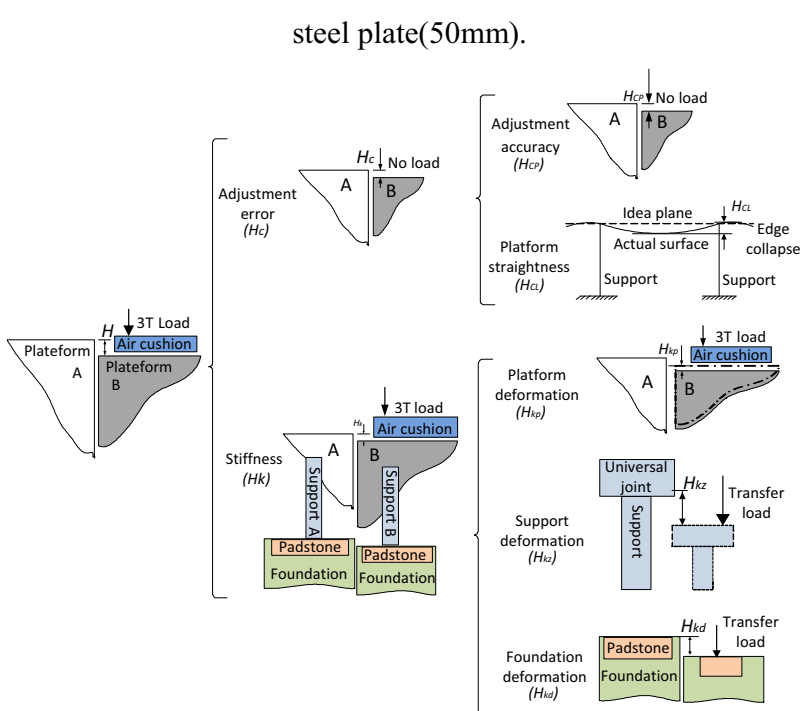

Figure 13. The diagram of decomposing mechanical index of altitude difference index

$$
H_{K D}=0.98 \mu m
$$

\subsubsection{The mechanical characteristics analysis of support}

there are more transmission structure, the elastic deformation of support is harder to get, so the index of support could be got by using residual quantity method, which is difference between the overall design index and deterministic subsystem index. The above index of subsystem will be summarized as follows:

(1) The index of adjustment accuracy

$$
H_{C P}=0.2 \mu m
$$

(2) The index of platform straightness

$$
H_{c L}=3.3 \mu m
$$

(3) The index of plateform elastic deformation

$$
H_{K P}=2.2 \mu m
$$

(4) The index of foundation elastic deformation

$$
H_{K D}=0.98 \mu m
$$

Using residual quantity method, the whole index of system is $10 \mu \mathrm{m}$, the sum of above four parts is $6.68 \mu \mathrm{m}$, so the index of support is $3.32 \mu \mathrm{m}$. It is based on thinking about heavy load deformation, adjustable accuracy and adjustment range. The support can satisfy requirements by investigating. The following equation 9 is established.

$$
H_{K Z}=3.32 \mu m
$$

Through above analysis, the altitude difference because of stiffness is $6.5 \mu \mathrm{m}$. Importing data into equation 6 , and the equation 10 is true.

$$
H_{K}=H_{K P}+H_{K Z}+H_{C D}=2.2+3.3+0.98=6.48 \mu m(10)
$$

Summarizing the figures of decomposing mechanical index as shown in Fig. 13.

\section{The analysis of whole system mechanical characteristics}

In summary, the whole model would be built by using Patran, the plate deformation of system would be analysed when the load is 3t. As shown in Fig. 14.

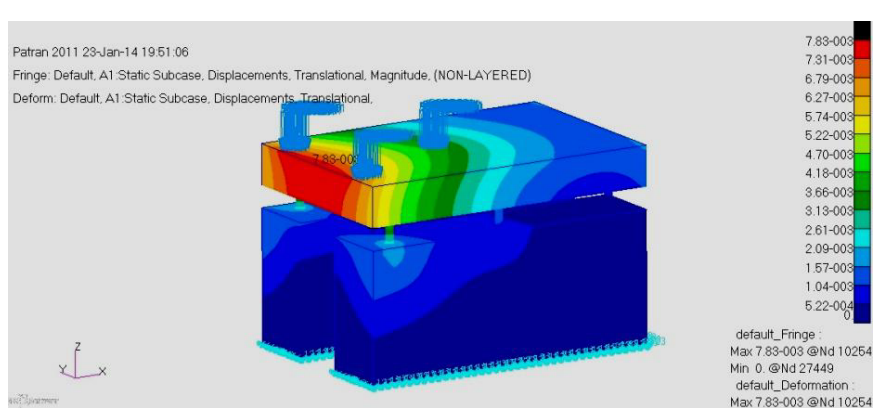

Figure 14. The deformation cloud diagram of whole system on 3 toad

When the load is $3 \mathrm{t}$, the levelness variation of plate is 0.5 " $<1$ ", the deformation of platform meet index requirements. The maximum stress of foundation is $0.96 \mathrm{MPa}$, the safety factor is greater than 200 .

\section{Summary}

This paper analyzes and researches the mechanical indexs (altitude difference) of The large supportive stage with super smooth surface. Affecting factors are divided into and adjustment error and system stiffness. First, analyzing adjustment accuracy and platform straightness, which influence the adjustment error. Second, analyzing platform mechanical characteristics、support mechanical characteristics and foundation mechanical characteristics, which influence the system stiffness. Conclusions are as follows:

(1) the index of motor adjustment accuracy is $0.2 \mu \mathrm{m}$;

(2) the index of platform straightness is $3.3 \mu \mathrm{m}$;

(3) the index of platform deformation is $2.2 \mu \mathrm{m}$;

(4) the index of foundation deformation is $0.98 \mu \mathrm{m}$;

(5) the index of support deformation is $3.32 \mu \mathrm{m}$;

(6) When the load is $3 \mathrm{t}$, the deformation of whole system is $9.79 \mu \mathrm{m}$, which is less than index requirement $10 \mu \mathrm{m}$.

Above index assignment and mechanics calculation are meet the technical requirements. The theoretical 
support and design method are provided in this paper, which is based on objective and reasonable considerations.

\section{References}

1. White G C, Xu Y S. Active Vertical-Gravity Compensation System[J].IEEE Transactions on Instr- umentation and Measurement,1994,43(6):786792.

2. J.S.Li, $\mathrm{Mu}$ X.G.Mu and J.J.Zhang. Physical Simulation for Satellite Control Systems[J]. Aerospace Control, 2004(4):37-43.

3. L.X.Xiao, Q.F.Jia and H.H.Deng: Theoretical Mechanics (Tianjin University press,1995).

4. N.M.Qi, W.H.Zhang, J.Z.Gao and M.Y.Huo, The Primary Discussion for the Ground Simulation
System of Spatial Microgravity[J]. Aerospace Control, 2011:95-100.

5. Y.S.Yao, T.Mei. Simulation Method of Space Operation on the Ground-Buoyancy Mehod[J]. Chinese Journal of Mechanical Engineering, 2008:182-188.

6. D. Miller,et al. SPHERES: A tested for long duration satellite formation flying in micro-gravityconditions [A]. Proceedings of the AAS/AIAA Space Flight Mechanics Meeting[C]. Clearwater, Florida, January 23-26,2000:167-179.

7. C.M.Lv, L.Xi, G.H.Zhao, Y.H.Zhang. Experiment Microgravity System for Zero Gravity Airplane[J].Journal of Tsinghua University(Science and Technology), 2003:1064-1068.

8. P.Zeng: Finite Element Analysis and Applications(Tsinghua University press,2004). 